\title{
Trends of Radiopharmaceutical Use at Mayo Clinic Rochester
}

Ashley A. Mroczenski, Stephanie M. Berent, Alice A. Hall, Joseph C. Hung, Thomas J. Herold, and Brian P. Mullan

Department of Radiology, Mayo Clinic, Rochester, Minnesota

The field of radiology is continuously changing. The purpose of this study was to identify the effect of technologic advances on nuclear medicine during the past $15 \mathrm{y}$. Methods: The number of radiopharmaceutical doses dispensed at Mayo Clinic (Rochester, Minnesota) from 1990 through 2004 was tracked. The number of doses was equivalent to the number of scans performed. Results: Since 1990, the number of bone scans decreased by $38 \%$. Brain scans using ${ }^{99 m} \mathrm{Tc}$ have increased by $166 \%$. The number of cardiac doses dispensed increased $184 \%$ from 1990 through 1999 but decreased 3\% between 2000 and 2004 . The number of lung scans decreased $52 \%$ from 1992 through 1999 and increased 66\% from 1999 through 2004. The number of kidney scans decreased $67 \%$ since 1990. Since its introduction in 1993, the use of ${ }^{111}$ In-pentetreotide has increased 16 -fold. PET data showed a $602 \%$ increase in the number of procedures from 2001 through 2004. Conclusion: The number of bone, lung, and kidney scans has decreased because of advances in other imaging modalities. Although the number of cardiac imaging scans increased during most of the study period, the recent rate of growth has declined, possibly because of the availability of alternative procedures such as stress echocardiography. The number of brain and lung scans performed has increased, partially because of the development of new protocols. PET and tumor imaging have shown a substantial increase because of increasing numbers of approved indications and Medicare reimbursement.

Key Words: nuclear pharmacy; radiopharmaceuticals; use trends

J Nucl Med Technol 2007; 35:154-158

DOI: $10.2967 /$ jnmt.106.038992

$\mathbf{T}$ he field of radiology is continuously changing. Knowledge of these changes and why they occurred will allow us to predict future use trends in nuclear medicine. Physicians order specific tests on the basis of the patient's symptoms and probable diagnosis. As technology advances, new tests are compared with previously established methods to determine the best method of diagnosing certain diseases. If the new test is superior, it may replace the

Received Dec. 15, 2006; revision accepted Apr. 2, 2007.

For correspondence contact: Joseph C. Hung, PhD, Department of Radiology, Mayo Clinic, 200 First St. SW, Rochester, MN 55905.

E-mail: jhung@mayo.edu

COPYRIGHT @ 2007 by the Society of Nuclear Medicine, Inc. previously used examination method. The purpose of this study was to identify changes in nuclear medicine use at Mayo Clinic (Rochester, Minnesota) over the past $15 \mathrm{y}$ and determine why those changes occurred.

\section{MATERIALS AND METHODS}

The number of radiopharmaceutical doses dispensed at Mayo Clinic Rochester from 1990 through 2004 was tracked using Nuclear Pharmacy Manager software (Bristol-Myers Squibb). This program tracks all doses dispensed from our laboratory, and the values correspond to the number of studies performed using a specific radiopharmaceutical agent. We determined the total number of scans performed each year and the number of scans performed using specific radiopharmaceuticals. Data for PET tumor imaging were based on the number of clinical scans performed each year, not the number of doses dispensed.

We measured the number of bone scans that used ${ }^{99 \mathrm{~m}} \mathrm{Tc}$ medronate and ${ }^{99 \mathrm{~m}} \mathrm{Tc}$-oxidronate; brain scans that used ${ }^{99 \mathrm{~m}} \mathrm{Tc}-$ exametazime and ${ }^{99 \mathrm{~m} T c-b i c i s a t e ; ~ m y o c a r d i a l ~ p e r f u s i o n ~ s c a n s ~ t h a t ~}$ used ${ }^{201} \mathrm{Tl}$-thallous chloride and ${ }^{99 \mathrm{~m} T c} \mathrm{~T}$-sestamibi; lung ventilation and perfusion scans that used ${ }^{133} \mathrm{Xe}$ gas and ${ }^{99 \mathrm{~m} T \mathrm{~T}-\text { macroaggre- }}$ gated albumin; kidney scans that used ${ }^{99 \mathrm{~m}} \mathrm{Tc}-$ mertiatide, ${ }^{99 \mathrm{~m}} \mathrm{Tc}-$ succimer (dimercaptosuccinic acid [DMSA]), ${ }^{99 \mathrm{~m} T c-p e n t e t a t e}$ (diethylenetriamine penta-acetic acid [DTPA]), and ${ }^{131}$ I-hippuran; and tumor scans that used ${ }^{111}$ In-pentetreotide and ${ }^{18} \mathrm{~F}$-FDG. These radiopharmaceuticals were chosen because of the prevalence of their use and because we suspected that their use patterns changed over time. Only radiopharmaceutical doses dispensed for clinical patients were included in our study (radiopharmaceuticals used in research studies were excluded). As new radiopharmaceuticals were developed, use of other pharmaceuticals typically diminished; older radiopharmaceuticals often were replaced completely by the new agents for imaging of a specific organ system. For example, ${ }^{99 \mathrm{~m}} \mathrm{Tc}-\mathrm{DTPA}$ was replaced by ${ }^{99 \mathrm{~m}} \mathrm{Tc}-$ mercaptoacetyltriglycine in renal scintigraphy. When more than one type of radiopharmaceutical was used for a particular imaging modality, values were compared and summed to determine the total number of scans of that organ system.

\section{RESULTS}

In 1990, approximately 30,000 doses were dispensed from the nuclear medicine laboratories of our institution. Since 2001, more than 50,000 doses have been dispensed annually (Fig. 1). Although the number of scans performed per year has increased, the use of each radiopharmaceutical has not increased proportionally to reflect that change. 


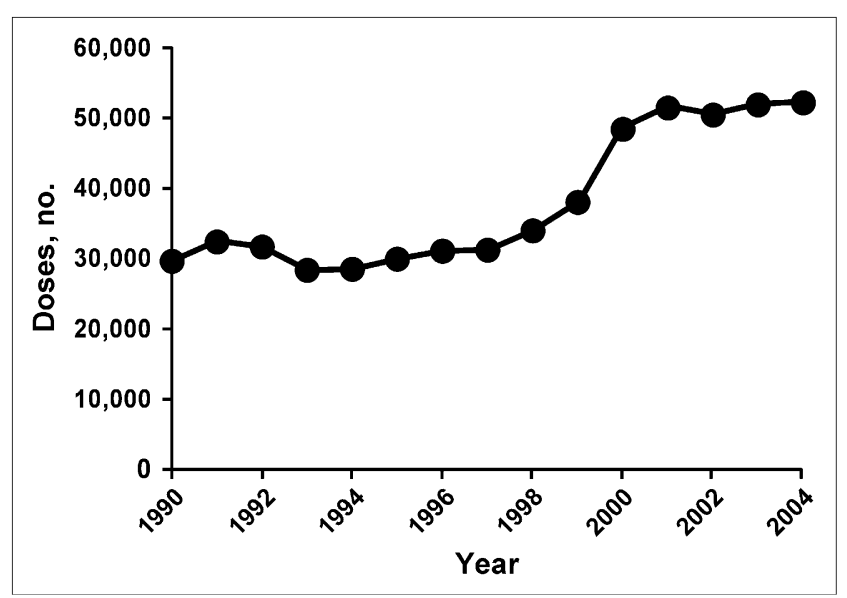

FIGURE 1. Total number of dispensed radiopharmaceutical doses.

Bone scan doses represented $30 \%$ of radiopharmaceuticals dispensed in 1990, but this percentage decreased to

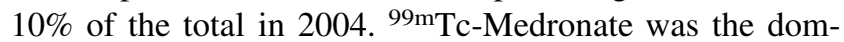
inant bone radiopharmaceutical used during the study period. ${ }^{99 \mathrm{~m}} \mathrm{Tc}$-Oxidronate was used almost exclusively in 1991 and 1992. Since 1990, the total number of bone scans decreased by $38 \%$ (Fig. 2). ${ }^{99 \mathrm{~m} T c-E x a m e t a z i m e ~ w a s ~ t h e ~}$ primary brain perfusion imaging agent from 1990 until 1994, when ${ }^{99 m}$ Tc-bicisate received U.S. Food and Drug Administration (FDA) approval for brain perfusion imaging. The number of brain scans performed at Mayo Clinic Rochester increased by 166\% between 1990 and 2004 (Fig. $3)$. These radiopharmaceuticals cross the blood-brain barrier and are used for patients with cerebrovascular disease, epilepsy, and Alzheimer's disease. In selected situations, physician-directed use of these radiopharmaceuticals has deviated from the indication on the package insert.

The number of dispensed cardiac doses increased by 184\% from 1990 until 1999 but decreased slightly (3\%) between 2000 and 2004 (Fig. 4). Starting in 1997, more

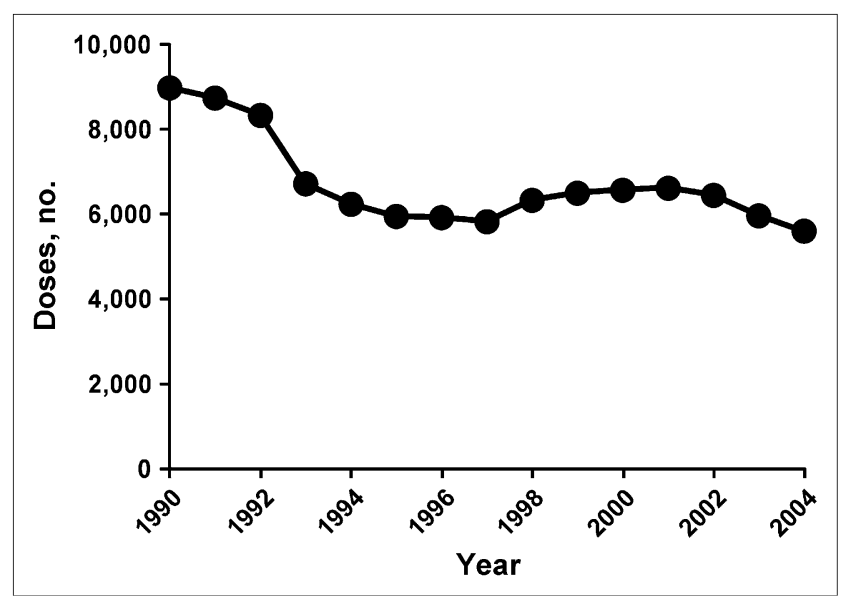

FIGURE 2. Radiopharmaceutical doses dispensed for bone scans.

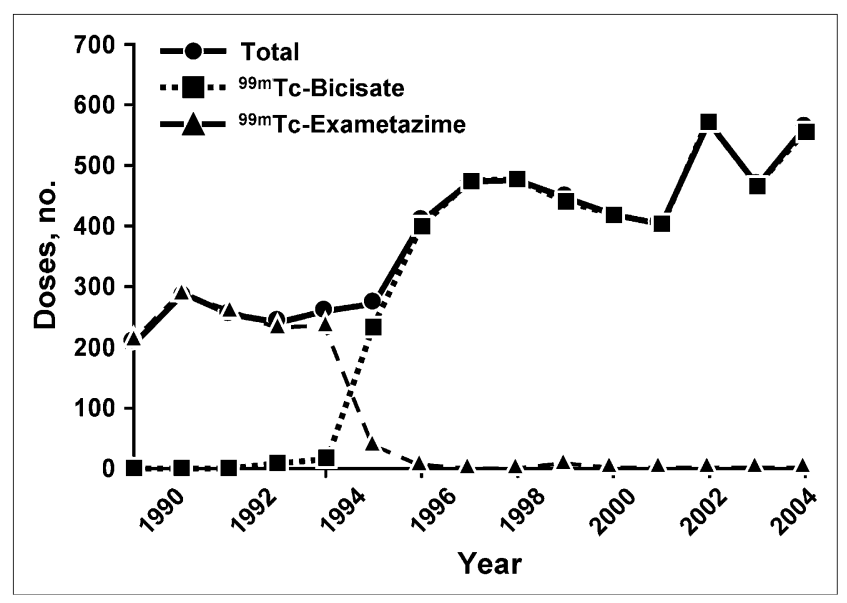

FIGURE 3. Performance of brain scans, stratified by radiopharmaceutical.

${ }^{99 \mathrm{~m}} \mathrm{Tc}$-sestamibi studies were performed than ${ }^{201} \mathrm{Tl}$-thallous chloride studies for myocardial perfusion imaging.

The number of lung scans decreased by $36 \%$ between 1990 and 1999 (Fig. 5). In 2004, 6\% more 99mTc-macroaggregated albumin doses were dispensed than in 1990, reflecting use of lung ventilation-perfusion scintigraphy in patients with supraventricular arrhythmias who were treated with right ventricular outflow tract ablation. From 1990 to 2004, the number of doses dispensed fluctuated.

The number of kidney scans decreased by $67 \%$ between 1990 and 2004 (Fig. 6). Four kidney imaging agents were used during this time: ${ }^{99 \mathrm{~m} T c-D T P A},{ }^{131} \mathrm{I}$-hippuran, ${ }^{99 \mathrm{~m}} \mathrm{Tc}-$ mertiatide, and $99 \mathrm{~m}$ Tc-DMSA. Renal scintigraphy has been replaced by ultrasonography and MRI for several previously common indications (e.g., assessment of renal artery stenosis). Currently, ${ }^{99 \mathrm{~m} T c-m e r t i a t i d e}$ and ${ }^{99 \mathrm{~m} T c-}$ DMSA are the only renal tracers frequently used at our institution.

Another increasingly used radiopharmaceutical is ${ }^{111} \mathrm{In}$ pentetreotide for neuroendocrine tumors. Our institution has

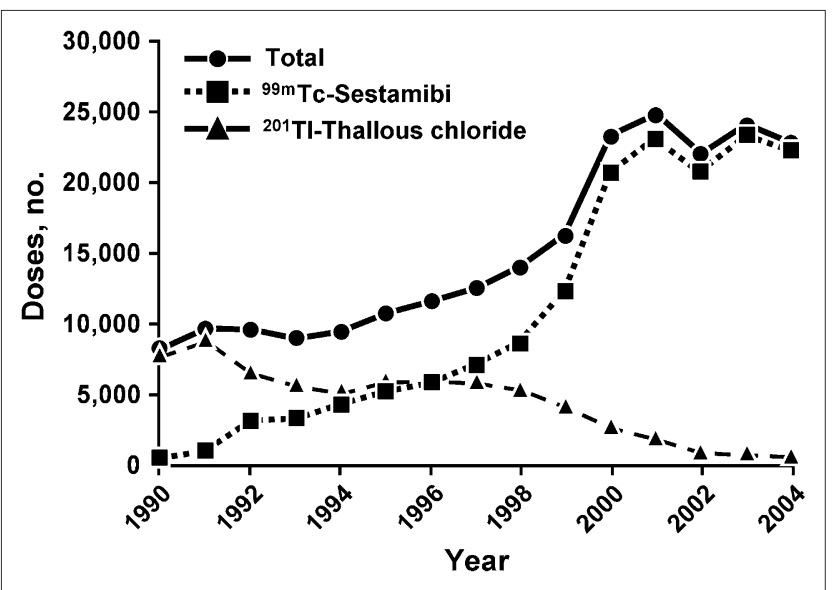

FIGURE 4. Radiopharmaceutical doses dispensed for cardiac scans. 


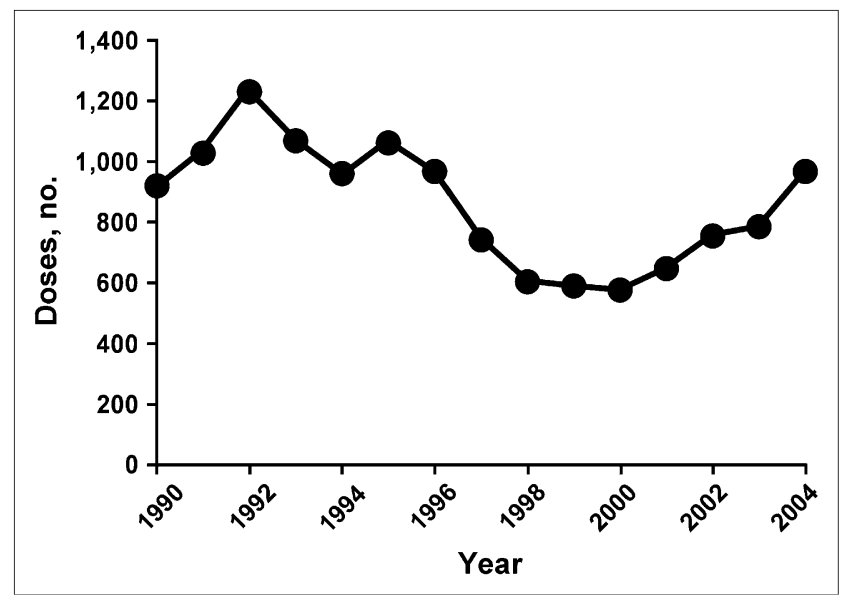

FIGURE 5. Radiopharmaceutical doses dispensed for lung scans.

seen a 16-fold increase in the amount of ${ }^{111}$ In-pentetreotide used since its introduction in 1993 (Fig. 7A). These relatively uncommon tumors are challenging to detect with conventional imaging when they are small; the increase in use likely is because patients are referred to Mayo Clinic Rochester for imaging.

PET imaging with ${ }^{18} \mathrm{~F}-\mathrm{FDG}$ began as a research protocol at Mayo Clinic Rochester in 1999 with the installation of the first PET scanner. Subsequent early use of ${ }^{18}$ F-FDG grew exponentially and showed a $602.1 \%$ increase in PET procedures from 2001 to 2004 (Fig. 7B). This increase also was facilitated by the later installation of 3 PET/CT scanners.

\section{DISCUSSION}

Over the study period, the use of bone scans decreased by $38 \%$ (Fig. 2). The largest decrease occurred in 1993. This downward trend corresponded to the discovery of the association between high levels of prostate-specific antigen (PSA) and the increased likelihood of having positive bone scan findings for patients with a new diagnosis of prostate

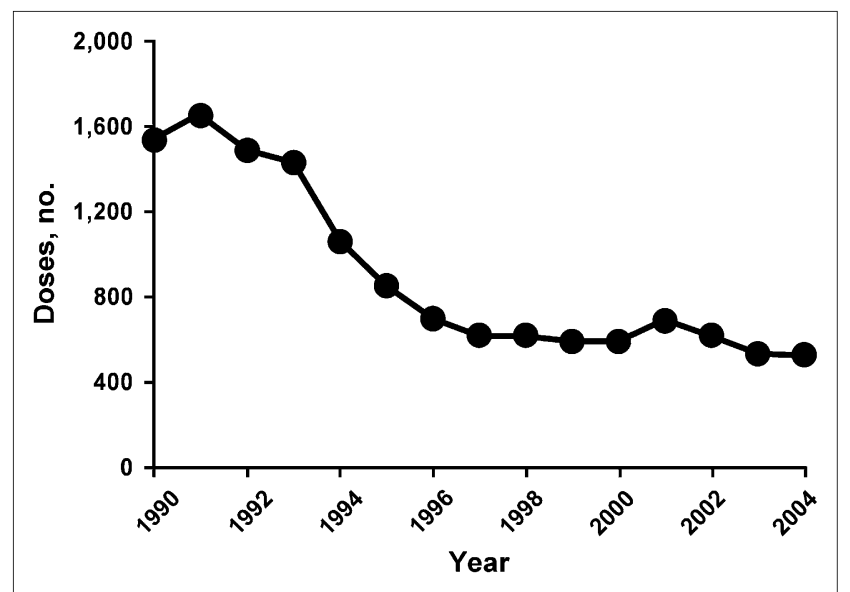

FIGURE 6. Radiopharmaceutical doses dispensed for kidney scans.

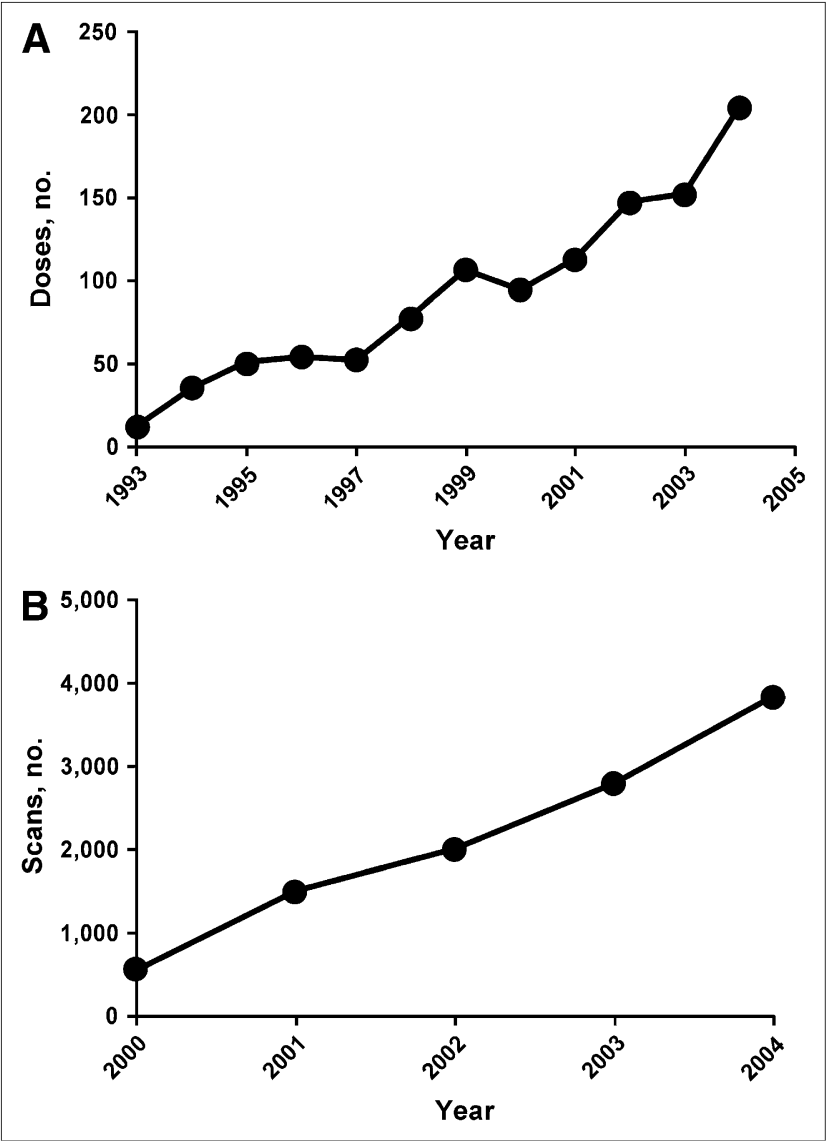

FIGURE 7. (A) ${ }^{111}$ In-Pentetreotide doses dispensed. (B) Radiopharmaceutical doses dispensed for PET scans.

carcinoma. According to Oesterling et al. (1), a radionuclide bone scan is unnecessary for a patient with no skeletal symptoms and a PSA level of less than $10.0 \mu \mathrm{g} / \mathrm{L}$. In addition, Dotan et al. (2) showed that trigger PSA, PSA velocity, and slope are associated with positive bone scan findings. They further suggested that a highly discriminative nomogram may be used to select patients according to their risk of having positive scan findings. PSA tests are easier and more cost-effective than bone scans for some patients. Currently, our physicians routinely use PSA tests for screening and order bone scans when necessary.

The number of brain scans increased $166 \%$ between 1990 and 2004 (Fig. 3) because of their diagnostic use for patients with cerebrovascular disease, epilepsy, or Alzheimer's disease. ${ }^{99 \mathrm{~m} T c-E x a m e t a z i m e}$ was the primary agent used for brain imaging until 1994 , when ${ }^{99 \mathrm{~m}} \mathrm{Tc}$-bicisate received FDA approval for use in perfusion imaging. ${ }^{99 \mathrm{~m}} \mathrm{Tc}-$ Bicisate was the preferred brain imaging agent at our institution because the early formulation of ${ }^{99 \mathrm{~m}} \mathrm{Tc}$-exametazime limits stability after reconstitution. Additionally, activity was higher if the agent could be reconstituted with a cold kit (3). The use of ${ }^{99 \mathrm{~m} T c}$-exametazime to evaluate patients with epilepsy has lower localization rates, poorer interobserver agreement, and less concordance with electroencephalogram 
findings, MRI findings, and discharge diagnosis than does the use of ${ }^{99 \mathrm{~m}}$ Tc-bicisate. ${ }^{99 \mathrm{~m}} \mathrm{Tc}$-Exametazime also produces higher uptake in the background extracerebral tissue, possibly because it clears more slowly from the bloodstream. Greater gray-white uptake differentiation is seen with ${ }^{99 m}$ Tc-bicisate, thus allowing subtle focal changes in the cortex to be detected more readily (3). When used for imaging of Alzheimer's disease, ${ }^{99 \mathrm{~m}} \mathrm{Tc}$-bicisate shows greater contrast between affected and unaffected brain structures (4).

The number of radiopharmaceutical doses dispensed for cardiac scans increased sharply (184\%) from 1990 through 1999 but decreased slightly (3\%) between 2000 and 2004 (Fig. 4). In 1997, more ${ }^{99 \mathrm{~m}}$ Tc-sestamibi doses were dispensed than ${ }^{201} \mathrm{Tl}$-thallous chloride doses for myocardial perfusion imaging. The use of ${ }^{99 \mathrm{~m}} \mathrm{Tc}$-sestamibi has continued to increase; it has essentially replaced ${ }^{201} \mathrm{Tl}$-thallous chloride for myocardial perfusion imaging. The superior imaging characteristics of ${ }^{99 \mathrm{~m}} \mathrm{Tc}$-sestamibi have made it the radiopharmaceutical of choice for Mayo Clinic Rochester.

Today, more than $40 \%$ of radiopharmaceutical doses dispensed at Mayo Clinic Rochester are used in the 2 nuclear cardiology laboratories. Although the number of myocardial perfusion scans is beginning to plateau, the ${ }^{99 \mathrm{~m}} \mathrm{Tc}$ sestamibi perfusion scan remains an important tool in the diagnosis of coronary artery disease (5). Although the exercise treadmill test is usually the first method used to assess cardiovascular function, the ${ }^{99 \mathrm{~m}} \mathrm{Tc}$-sestamibi scan often is performed if the exercise treadmill test findings are inconclusive or indicate possible ischemic changes. At our institution, the choice of test often depends on the patient's condition. For example, if the physician is concerned about blood perfusion to the myocardium, a ${ }^{99 \mathrm{~m}} \mathrm{Tc}$-sestamibi scan is the best choice. However, if valve function is the main concern, an echocardiogram is a better option. Studies with ${ }^{99} \mathrm{~m}$ Tc-sestamibi may be adapted easily to pharmacologically induced stress (e.g., by using dobutamine or adenosine) for patients who are unable to exercise. Stress echocardiography is limited to the use of dobutamine pharmacologic stress when exercise is not feasible.

The slight decrease in the number of ${ }^{99} \mathrm{~m}$ Tc-sestamibi perfusion scans performed in recent years is attributable to the increased use of stress echocardiography for patients with a clinical suspicion of coronary artery disease. Although cardiac PET has been introduced to our laboratory, its use remains limited.

The number of lung scans performed decreased by $52 \%$ between 1992 and 1999 (Fig. 5). The primary reason for the decline in lung ventilation and perfusion imaging is the development of spiral volumetric CT for detection of pulmonary embolism. According to van Rossum et al. (6), spiral volumetric CT for pulmonary embolism has a sensitivity of $95 \%$ and a specificity of $97 \%$; the positive and negative predictive values are $97 \%$ and $97 \%$, respectively. Additionally, breathing artifacts are problematic in nuclear lung ventilation and perfusion imaging studies but do not occur with CT (7). However, a steady increase (66\%) in the number of lung scans occurred between 1999 and 2004 (Fig. 5). This increase was due to the use of a protocol that evaluates the effect of pulmonary vein ablation therapy on surrounding lung tissue. Right ventricular outflow tract ablation therapy is used for patients with supraventricular arrhythmias or atrial arrhythmia. A lung ventilation and perfusion scan is performed before ablation and again 3-6 mo after ablation.

The use of kidney scans has decreased by $67 \%$ since 1990 (Fig. 6). Four kidney imaging agents were used during that time: ${ }^{99 \mathrm{~m}} \mathrm{Tc}-\mathrm{DTPA},{ }^{131} \mathrm{I}$-hippuran, ${ }^{99 \mathrm{~m}} \mathrm{Tc}-\mathrm{mertiatide}$, and ${ }^{99 m}$ Tc-DMSA. In 1990, ${ }^{99 m}$ Tc-DTPA and ${ }^{131}$ I-hippuran were the kidney imaging agents used most frequently at Mayo Clinic Rochester. ${ }^{99 \mathrm{~m}} \mathrm{Tc}-\mathrm{DTPA}$ is removed from the kidneys by glomerular filtration and requires only a small dose because the process is slow. ${ }^{131}$ I-Hippuran is cleared from the kidneys mostly by tubular secretion but has poor imaging characteristics. These agents were replaced by ${ }^{99 \mathrm{~m}}$ Tc-mertiatide. ${ }^{99 \mathrm{~m}}$ Tc-Mertiatide leaves the kidneys by tubular secretion, yielding superior imaging characteristics for determining kidney function. For imaging the size, shape, and position of the kidneys, ${ }^{99 \mathrm{~m} T c-D M S A}$ is preferred because it accumulates in the kidneys ( 8 ).

The decreased use of renal scanning is due to advances in other imaging modalities. The diagnostic standard for determining renal artery stenosis is renal angiography, but this procedure is invasive and expensive. Duplex ultrasonography and magnetic resonance angiography are replacing captopril renography (9). One study suggested that duplex ultrasonography and captopril renography have high specificity and negative predictive value for detecting renal artery stenosis but also indicated that duplex ultrasonography is easier to perform and is more cost effective (10). Similarly, another study showed that duplex ultrasonography is widely available and provides anatomic and functional information (11). Duplex Doppler ultrasonography for renal artery stenosis greater than $50 \%$ has a sensitivity of $97 \%$ and specificity of $98 \%$ (12). Magnetic resonance angiography may provide more anatomic information than duplex ultrasonography and has a sensitivity of $100 \%$ and a specificity of $94 \%$ (13).

Another radiopharmaceutical with increasing use is ${ }^{111}$ In-pentetreotide. Mayo Clinic Rochester had a 16-fold increase in the amount of ${ }^{111}$ In-pentetreotide dispensed since its introduction in 1993 (Fig. 7A). The initial increase occurred after U.S. FDA approval was obtained in 1994. The use of ${ }^{111}$ In-pentetreotide continued to rise as the cost decreased and availability increased. Another contributing factor has been the use of SPECT and CT. The merging of these 2 modalities provides physicians with valuable information that aids in patient diagnosis and care.

The use of PET at Mayo Clinic Rochester began as a research protocol in 1999. In 1997, the FDA Modernization and Accountability Act was issued to help regulate PET drugs, which were already being produced and used in academic institutions and hospitals (14). Since 1999, the number of 
scans performed has continued to increase, particularly in 2001, when use of ${ }^{18}$ F-FDG for the evaluation of certain tumors was approved for Medicare reimbursement. Our institution performed 3 clinical scans in 2000 and 76 in 2001. The increase in the number of PET procedures between 2001 and 2004 was 602\% (Fig. 7B). This increase was attributable directly to the FDA decision to expand Medicare coverage of PET scans to include the diagnosis, staging, and restaging of non-small cell lung cancer, esophageal cancer, colorectal cancer, lymphoma, melanoma, and head and neck cancers; evaluation of myocardial viability after an inconclusive SPECT scan; and presurgical evaluation of refractory seizures (14). As the number of indications approved for Medicare reimbursement increased, the number of PET scans performed at Mayo Clinic Rochester also increased, causing a marked change in our nuclear medicine department. Initially, the department had only 1 PET scanner, but 3 more have been acquired since then ( 2 are PET/CT scanners). Advances in PET scanner technology and the introduction of PET/CT have been contributing factors in the growth of PET for patient care. Although Mayo Clinic Rochester uses other PET radiopharmaceuticals, only ${ }^{18}$ F-FDG use was considered for this study.

\section{CONCLUSION}

We expect the use of PET and molecular imaging to continue to grow. Nuclear medicine will change and grow as new test procedures are developed for other imaging modalities and as our own technology improves. Although the numbers of some procedures have decreased, the annual number of nuclear medicine doses dispensed at Mayo Clinic Rochester has increased slightly during the study period. Many current procedures use SPECT and PET to acquire high-quality images. On the basis of changes that occurred during the past $15 \mathrm{y}$, we expect to incorporate more molecular imaging techniques in the future.

\section{ACKNOWLEDGMENTS}

Editing, proofreading, and reference verification were provided by the Section of Scientific Publications, Mayo Clinic. This study was presented at the Society of Nuclear Medicine Annual Meeting, San Diego, California, June 3-7, 2006.

\section{REFERENCES}

1. Oesterling JE, Martin SK, Bergstralh EJ, Lowe FC. The use of prostate-specific antigen in staging patients with newly diagnosed prostate cancer. JAMA. 1993;269:57-60.

2. Dotan ZA, Bianco FJ Jr, Rabbani F, et al. Pattern of prostate-specific antigen (PSA) failure dictates the probability of a positive bone scan in patients with an increasing PSA after radical prostatectomy. J Clin Oncol. 2005;23:19621968.

3. O'Brien TJ, Brinkmann BH, Mullan BP, et al. Comparative study of ${ }^{99 \mathrm{~m}} \mathrm{Tc}-\mathrm{ECD}$ and $99 \mathrm{mTc}-\mathrm{HMPAO}$ for peri-ictal SPECT: qualitative and quantitative analysis. J Neurol Neurosurg Psychiatry. 1999;66:331-339.

4. van Dyck $\mathrm{CH}$, Lin $\mathrm{CH}$, Smith EO, et al. Comparison of technetium-99mHMPAO and technetium-99m-ECD cerebral SPECT images in Alzheimer's disease. J Nucl Med. 1996;37:1749-1755.

5. Tang WH, Maroo A, Young JB. Ischemic heart disease and congestive heart failure in diabetic patients. Med Clin North Am. 2004;88:1037-1061.

6. van Rossum AB, Treurniet FE, Kieft GJ, Smith SJ, Schepers-Bok R. Role of spiral volumetric computed tomographic scanning in the assessment of patients with clinical suspicion of pulmonary embolism and an abnormal ventilation/ perfusion lung scan. Thorax. 1996;51:23-28.

7. Dresel S, Stabler A, Scheidler J, Holzknecht N, Tatsch K, Hahn K. Diagnostic approach in acute pulmonary embolism: perfusion scintigraphy versus spiral computed tomography. Nucl Med Commun. 1995;16:1009-1015.

8. Royal HD. Genitourinary system. In: Christian PE, Bernier DR, Langan JK, eds. Nuclear Medicine and PET: Technology and Techniques. 5th ed. St. Louis, MO: Mosby; 2004:481-482.

9. Olin JW. Renal artery disease: diagnosis and management. Mt Sinai J Med. 2004;71:73-85.

10. Johansson M, Jensen G, Aurell M, et al. Evaluation of duplex ultrasound and captopril renography for detection of renovascular hypertension. Kidney Int. 2000;58:774-782.

11. Karasch T, Rubin J. Diagnosis of renal artery stenosis and renovascular hypertension. Eur J Ultrasound. 1998;7(suppl 3):S27-S39.

12. Radermacher J, Haller $H$. The right diagnostic work-up: investigating renal and renovascular disorders. J Hypertens Suppl. 2003;21:S19-S24.

13. Davidson RA, Wilcox CS. Newer tests for the diagnosis of renovascular disease. JAMA. 1992;268:3353-3358.

14. Keppler JS. Federal regulations and reimbursement for PET. J Nucl Med Technol. 2001;29:173-179. 